\title{
Protocolo experimental para la evaluación de microemulsiones que modifican la mojabilidad en el medio poroso. Aplicación a campos colombianos
}

\author{
Manuel G. Jaimes ${ }^{1 *}$; Jorge A. Rojas ${ }^{1}$; Milton J. Rodríguez ${ }^{1}$; Richard D. Zabala ${ }^{1}$; Ricardo Dorado ${ }^{2}$ \\ ${ }^{1}$ Ecopetrol S.A. \\ ${ }^{2}$ Universidad Industrial de Santander \\ Código Postal: 681011. Piedecuesta - Colombia \\ Email: *Manuel.Jaimes@ecopetrol.com.co
}

\begin{abstract}
Resumen
Las reservas de crudo liviano son cada vez más escasas a diferencia de los descubrimientos de crudo pesado, razón por lo cual se ha enfocado la atención en este tipo de hidrocarburos.

Colombia tiene un gran potencial de crudo pesado, sobre todo en las zonas de la Cuenca de los Llanos Orientales (campos: Rubiales, Castilla, Chichimene, Apiay, Suria), cuyo desarrollo en los últimos años ha logrado que el 60\% de la producción nacional este asociada a este tipo de recurso.

La producción de crudo pesado no es fácil, una de las principales dificultades es la alta viscosidad del hidrocarburo, lo cual origina una baja movilidad del mismo y una temprana producción de agua en yacimientos con acuíferos activos.

Una de las técnicas más utilizadas para incrementar la productividad en los yacimientos de crudos pesados es la estimulación matricial de pozos, a partir de la cual se pueden modificar las curvas de permeabilidad relativa (alteración de la humectabilidad) y así mejorar la movilidad de estos crudos. Sin embargo, es crucial la comprensión de la mojabilidad de la formación para optimizar la recuperación de petróleo. Suponer que una formación es mojable por agua, cuando en realidad no lo es, puede ocasionar daños irreversibles en el yacimiento.
\end{abstract}

Por lo anterior, el presente estudio se centró en desarrollar e implementar un protocolo a nivel de laboratorio, para evaluar tratamientos de estimulación matricial base agua, que tuvieran la capacidad de invertir la mojabilidad natural (generalmente al crudo, debido a la alta afinidad entre el crudo y minerales de roca del yacimiento), hacia una mojabilidad al agua, que conduce a un mejoramiento de la permeabilidad relativa al crudo.

El protocolo experimental fue implementado a nivel de laboratorio en el campo Castilla (cuenca de los llanos orientales en Colombia) y se enfocó en tres grandes etapas.

1. Etapa 1: Caracterización básica de fluidos de formación

2. Etapa 2: Evaluación fluido-fluido y comportamiento reológico de fluidos de formación Vs Tratamientos químicos.

3. Etapa 3: Evaluación del efecto sobre la humectabilidad (modificación de ángulos de contacto y permeabilidad relativa).

Las principales conclusiones y recomendaciones obtenidas en este estudio son las siguientes:

1. Se desarrolló un protocolo experimental adecuado para la evaluación de microemulsiones y/o aditivos mejoradores de movilidad.

2. La implementación del protocolo permitió seleccionar el mejor tratamiento de alteración de la humectabilidad para el campo Castilla.

Cita: Jaimes, M. G., Rojas, J. A., Rodríguez, M. J., Zabala, R. D. y Dorado, R. (2019). Protocolo experimental para la evaluación de microemulsiones que modifican la mojabilidad en el medio poroso. Aplicación a campos colombianos. Revista Fuentes: El reventón energético, $17(2), 63-75$. 
3. En la preferencia de la mojabilidad del yacimiento, tienen incidencia: los componentes del petróleo, la química de la salmuera, la superficie mineral, la temperatura, la presión, la tensión interfacial de los fluidos, la presión capilar y la historia de saturación del yacimiento.

Palabras claves: acuífero activo, ángulo de contacto, daño de formación, humectabilidad, microemulsiones, mojabilidad, movilidad, permeabilidad relativa, presión capilar, productividad de pozo, tensión interfacial, viscosidad.

\title{
Experimental Protocol for Evaluation of Micro-Emulsions That Modify the Mojability in Porous Media. Application to Colombian Fields
}

\begin{abstract}
Reserves of light crude oil are decreasing in contrast to the discoveries of heavy crude, which is why attention has been focused on this type of hydrocarbons.

Colombia has a great potential for the heavy-crude development, especially in the areas of the Llanos Orientales Basin (campos: Rubiales, Castilla, Chichimene, Apiay, Suria), whose development in recent years has achieved the $60 \%$ of the national production to be associated with this type of resource.

Heavy-oil production is not an easy task due to its high viscosity, which causes low oil mobility and early water production in reservoirs with active aquifers.

One of the most used techniques to increase productivity in heavy crude oil fields is the matrix stimulation of wells through the injection of treatments that positively alter the relative permeability curves (alteration of wettability), and thus improve the mobility of these crude oils. However, understanding the formation wettability is essential to optimize the oil recovery. To assume that a formation is wettable by water, when in fact it is not, can cause irreversible damage to the reservoir.

Therefore, the current study developed and implemented a laboratory-level protocol to evaluate water-based matrix stimulation treatments that have ability to reverse natural oil wettability (generally to the oil, due to high affinity between oil and rock minerals of reservoir) towards water wettability, which leads to an improvement of relative permeability to crude oil.
\end{abstract}

A laboratory-level protocol was implemented in the Castilla Oilfield (Llanos Orientales basin in Colombia) and focused on three major stages.

1. Stage 1: Basic characterization of treatment fluids.

2. Stage 2: Fluid-fluid evaluation and rheological behavior of formation fluids Vs. Chemical treatments.

3. Stage 3: Evaluation of effect on wettability (modification of contact angles and relative permeability).

The main conclusions and recommendations of this study are:

1. A suitable experimental protocol was developed for evaluation of microemulsions and / or mobility enhancing additives.

2. Implementation of protocol allowed selecting the best treatment of alteration of wettability for the Castilla oilfield.

3. The components of the oil, the brine chemistry, the mineral surface, the temperature, the pressure, the interfacial tension, the capillary pressure, the saturation history of reservoir, among other properties have an impact on the reservoir's wettability.

Keywords: active aquifer, contact angle, formation damage, humidity, microemulsions, mojability, mobility, relative permeability, capillary pressure, well productivity, interfacial tension, viscosity.

\section{Introducción}

El incremento de demanda de energía de hidrocarburo, las condiciones cada vez más difíciles para encontrar crudo liviano y la necesidad de restituir reservas, han impulsado a la industria petrolera a explotar las reservas de crudo pesado y extrapesado que han llegado a constituir el $70 \%$ del total de las reservas de crudo a nivel mundial; la cual oscila entre 9 y 13 trillones de barriles, véase Figura 1. Por lo anterior, se espera que la producción mundial de petróleo pesado aumente entre 2 y 3 millones de barriles por día, lo que representa un crecimiento del 44\% en los próximos 10 años (Jaimes, Pachón, Villar, Dorado, 2011). 


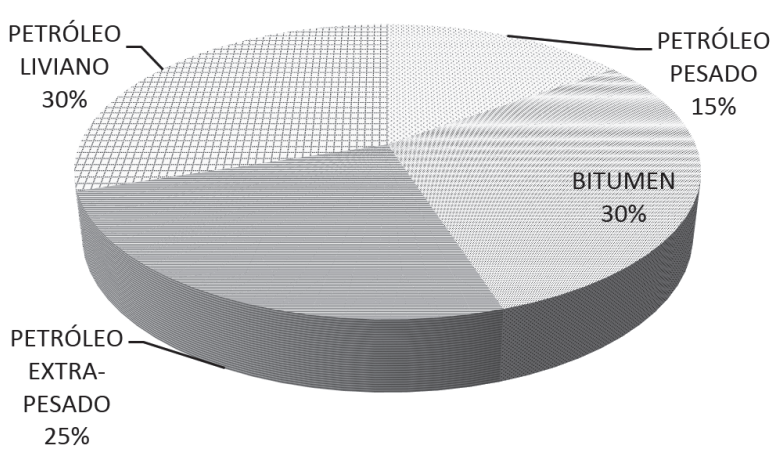

Figura 1. Reservas por tipo de crudo. Fuente: Oilfield Review 2009.

En Colombia existen 7 cuencas en las cuales hay campos de hidrocarburos. Estas cuencas son: Caguan - Putumayo, Catatumbo, Cordillera Oriental, Llanos Orientales, Valle Bajo, Medio y Superior del Magdalena, en las cuales existen diferentes tipos de crudos que van desde $63^{\circ}$ API (Campo de Cerro Gordo), hasta 0,92 ${ }^{\circ}$ API (campo de Valdivia-Almagro), pero el petróleo es mayormente pesado y extra pesado, entre 13 y 10 grados API, y más del $60 \%$ de las áreas con prospectividad tienen esa característica.

La Cuenca de los Llanos Orientales es la principal fuente de la industria petrolera en Colombia, ya que aporta el $75 \%$ de la producción nacional, véase Figura 2.

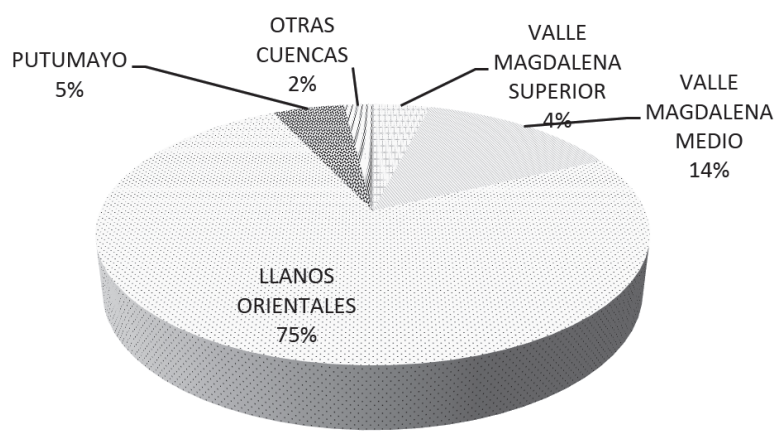

Figura 2. Producción de crudo, Colombia - 2015 [999 kbpd]. Fuente: ANH, 2015.

Este activo nacional está constituido de forma relevante por crudo pesado, el cual contribuye al $30 \%$ de incorporación de nuevas reservas y al $50 \%$ de los 2.002 millones de barriles de reservas probadas que tenía certificadas el país, a diciembre de 2015 (Asociación Colombiana del Petróleo, 2015). Por lo tanto, en los últimos años se han venido desarrollando reservas de petróleo pesado, sobre todo en los campos Rubiales, Castilla, Chichimene, Apiay y Suria.
En 2014 la producción de crudo pesado en Colombia correspondía al $60 \%$ de la producción nacional.

\section{Caracterización del problema}

En la producción de crudos pesados la problemática se enfoca principalmente en:

1. Geología poco propicia, donde las zonas que contienen los hidrocarburos se encuentran conectados con acuíferos activos.

2. La mojabilidad generalmente al crudo que presentan los yacimientos, debido a la alta afinidad entre el crudo pesado y minerales de roca del yacimiento.

3. La naturaleza propia del crudo pesado, la cual presenta baja gravedad API y alta viscosidad, que representan una relación de movilidad desfavorable del crudo, provocando tempranamente altos cortes de agua en yacimientos con acuíferos activos.

4. Daños de formación generados durante la etapa de perforación, completamiento y producción que favorecen la movilidad del agua.

La coyuntura actual de los precios del petróleo, con cada vez mayor volatilidad, ha afectado también el segmento de los crudos pesados en Colombia. Mientras en 2000 la producción de este tipo de petróleo apenas llegaba al $10 \%$, en la actualidad supera el $60 \%$. "Esto, además de plantear un reto desde el punto de vista de costos de producción, sugiere un desafío porque la tarifa es más baja de la que se paga por crudos livianos"

Por lo anterior, se hace necesaria la incorporación de tecnología que permita mejorar la movilidad del crudo pesado en el yacimiento, incrementar la producción de crudo, disminuir la producción de agua, generar ahorros en el costo de producción de barril producido e incrementar el factor de recobro.

\section{Solución al problema}

Hay muchas tecnologías probadas con éxito frente al reto que exigen los crudos pesados y que les han dado la competitividad en el segmento de producción. Entre estas tecnologías se encuentran: Tecnologías de estimulación, recobro térmico por inyección de vapor al yacimiento (Díaz, Navarro \& Tavera, 2007), combustión in-situ (Trujillo, Delgadillo, Claro, Rojas \& Sandoval, 2018), pozos horizontales (Sierra, Navarro \& Tavera, 2006), bombas electro sumergibles (Camacho \& Grosso, 2003) y muchas otras tecnologías. 
Este estudio se enfocó en el desarrollo e implementación de protocolos que permitan evaluar tecnologías de estimulación a nivel de laboratorio, cuyo objeto sea mejorar la movilidad de crudos pesados.

Los tratamientos de estimulación matricial objeto de este estudio, son micro emulsiones (ME) y/o aditivos mejoradores de movilidad (AMM) base agua (Salager, Antón \& Aubry, 2001), los cuales deben tener la capacidad de invertir la mojabilidad natural (generalmente al crudo, debido a la alta afinidad entre el crudo y minerales de roca del yacimiento), hacia una mojabilidad al agua, que conduce a un mejoramiento de la permeabilidad relativa al crudo (Kro) y a una disminución de la permeabilidad relativa al agua (Krw) (Meneses, Moreno \& Plata, 2017).

Para escoger el tratamiento de ME y/o AMM adecuado, es necesario el uso de un protocolo riguroso a nivel de laboratorio, que abarque primero la evaluación fluidofluido y fluido roca de las principales propiedades a impactar en el medio poroso, y segundo que tenga la capacidad de predecir un resultado atractivo desde el punto de vista técnico.

\section{Campo de estudio}

Como campo de estudio se seleccionó el campo Castilla, el cual está localizado en la cuenca de los Llanos orientales. Este campo se encuentra ubicado en el departamento del Meta, aproximadamente $54 \mathrm{Km}$ al sur de la ciudad de Villavicencio, en inmediaciones de los municipios de Castilla La Nueva y Acacias (Figura 3).

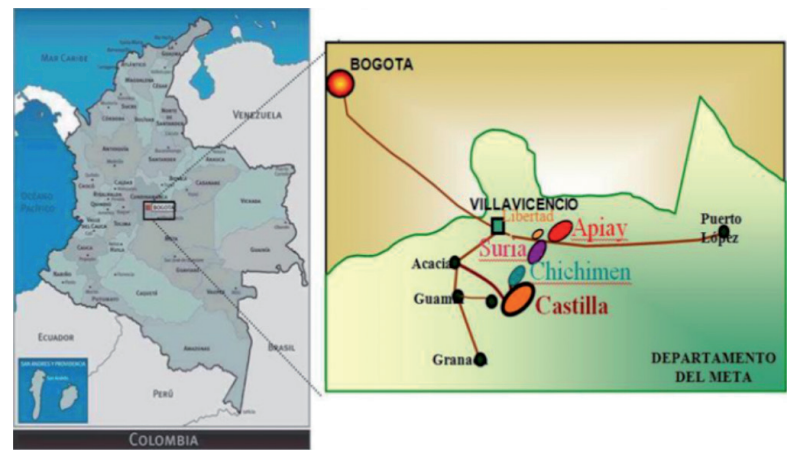

Figura 3. Ubicación campo Castilla. Fuente: Ecopetrol.

El campo Castilla se encuentra dividido en tres áreas operacionales: Campo Castilla, Campo Castilla Norte y Campo Castilla Este, no obstante todas pertenecen a la misma estructura geológica. Para septiembre de 2017 el campo contaba con 586 pozos productores perforados distribuidos así: 243 pozos en Castilla
Norte, 343 pozos en Castilla Sur y 1 pozo en Castilla Este. La producción promedio del campo a agosto de 2017 era de 114.000 BOPD con un corte de agua del $92 \%$. La producción acumulada de crudo y agua al 30 de septiembre del 2017 era de 568 MBls y 2,835 MBls, respectivamente (Figura 4).

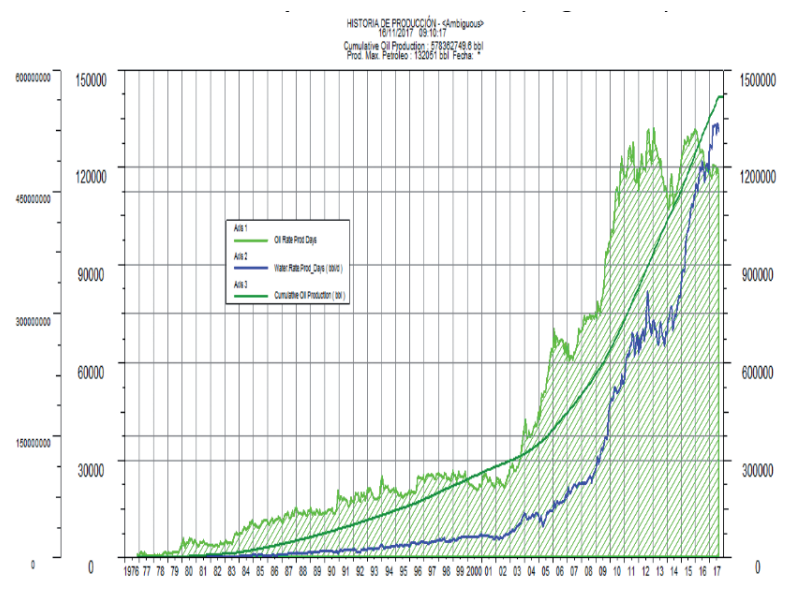

Figura 4. Producción diaria (bbl/día) y acumulada de agua y crudo campo Castilla. Fuente: Ecopetrol.

La sección estratigráfica del Bloque Cubarral se encuentra formada por rocas sedimentarias cuyas edades varían desde el paleozoico hasta el Cuaternario. El espesor total de la columna litológica alcanza los 18.000 pies. Las rocas sedimentarias de edad Cretácea y Terciaria son los principales yacimientos en el área. Las formaciones productoras son: Une (Unidad K2), Chipaqué (Unidad K1). La Figura 5 muestra respectivamente la columna estratigráfica.

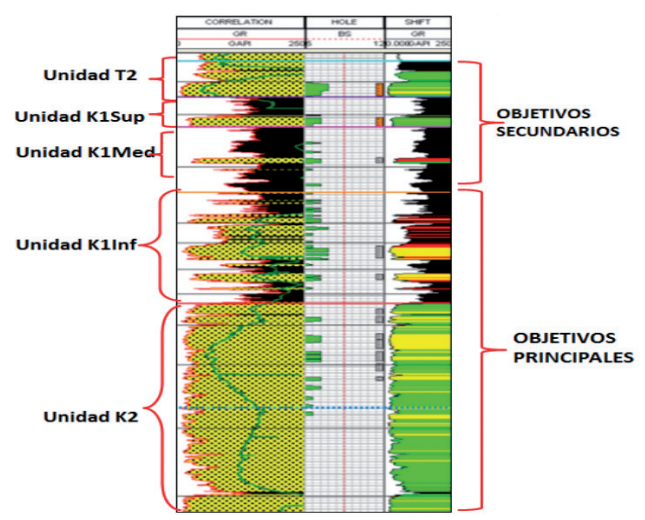

Figura 5. Columna estratigráfica campo Castilla. Fuente: Ecopetrol.

\section{Fundamentos teóricos}

Antes de describir el protocolo desarrollado, conviene revisar algunos fundamentos teóricos o conceptuales para comprender mejor el protocolo experimental diseñado. 


\section{Sistemas crudo-agua-surfactante}

Los crudos pesados son altamente viscosos por su alta concentración y agregación de asfaltenos (Martínez, de Lourdes, Zapata, Mar, Bernal, de la Cruz \& Aburto, 2011; Sánchez, Sánchez, González \& Ramírez, 2017) y por la incorporación de agua en forma de emulsión (W/O).

Para tratar este problema se utilizan tratamientos químicos (Guerrero, Montes, Oliveira, Campos \& Lucas, 2018), los cuales actúan como reductores de viscosidad base crudo o base agua y como modificadores de la permeabilidad relativa (incremento de la Kro y disminución de la Krw). Estos últimos corresponden a agentes surfactantes que modifican el comportamiento de la mezcla agua/crudo (Figueira, Simão, Soares \& Lucas, 2017).

Así, un determinado surfactante o mezcla de surfactantes presentará la funcionalidad de reductor de viscosidad, rompedor de emulsión, modificador de mojabilidad o un efecto adverso según las condiciones fisicoquímicas de aplicación, por lo cual es importante determinar el rango en el cual se obtiene el desempeño deseado (García, Rodríguez, Casallas, Cruz, Hernández, Afanador \& Rodríguez, 2010).

\section{Fundamentos de Mojabilidad}

La comprensión de la mojabilidad de la formación es crucial para optimizar los procesos de estimulación de pozos (el hecho de que sea mojable por crudo o por agua incide en el desempeño del yacimiento). Suponer que una formación es mojable por agua, cuando en realidad no lo es, puede ocasionar daños irreversibles en el yacimiento.

El término mojabilidad describe la preferencia de un sólido por estar en contacto con un fluido en lugar de otro. El termino preferencia describe adecuadamente el equilibrio de las fuerzas superficiales e interfaciales. Habitualmente, los constituyentes primarios de los yacimientos - cuarzo, carbonato y dolomía son mojables por agua antes de la migración del petróleo.

Algunos tratamientos de estimulación están diseñados para alterar la preferencia de la mojabilidad de la formación o bien a reducir la tensión interfacial existente entre los fluidos, reduciendo de ese modo las fuerzas de mojabilidad.

\section{Cambios en la mojabilidad}

Las fuerzas de mojabilidad conducen a una condición de equilibrio entre al menos tres sustancias: un sólido y dos fluidos. Los constituyentes y las condiciones para las tres sustancias inciden en la preferencia de la mojabilidad. Por lo tanto, debemos considerar los componentes del petróleo, la química de la salmuera, y la superficie mineral, así como también la temperatura, la presión y la historia de saturación del sistema (Martin \& Páez, 2017).

- Alteración de la mojabilidad debido a los asfaltenos y las fracciones polares del crudo. Cuando la superficie de la roca entra en contacto con el crudo, hay la posibilidad de modificar su superficie debido a la adsorción de asfaltenos.

- Influencia de la mojabilidad sobre la presión capilar. Para incrementar la recuperación de petróleo, se hace necesario la modificación de las fuerzas capilares por cambio de mojabilidad, reducción de la tensión interfacial o incluso eliminación de la interfase que es equivalente a alcanzar una tensión nula (miscibilidad de los fluidos), lo cual podría lograrse con concentración de surfactante del orden de 5\%. En tales condiciones se producen varios mecanismos de movilización que contribuyen a la recuperación del crudo.

- Efecto de la mojabilidad sobre las permeabilidades relativas. Esta propiedad de flujo es el resultado de los efectos de la geometría de los poros, de la mojabilidad, de la distribución de los fluidos y de la historia de saturación. Las mediciones en laboratorio corresponden a las reales de la formación únicamente si la preferencia de mojabilidad de la muestra en el laboratorio es la misma que en la formación.

- Comportamiento de las permeabilidades relativas en sistemas de mojabilidad homogénea. Para analizar los efectos de la mojabilidad sobre las permeabilidades relativas en estos tipos de sistemas, la mojabilidad de toda la superficie se restaura de mojada por agua a mojada por petróleo, manteniéndose en cada caso lo más uniforme posible. En general, a cualquier saturación, la permeabilidad relativa a un fluido es alta cuando este es el fluido no mojante.

- Efecto de la depositación de sales. La depositación de una fina capa de partículas sobre las superficies de cuarzo, generó una permanente tendencia de la superficie a estar fuertemente mojada por agua (observación por medición de ángulos de contacto). 
- Variación de la mojabilidad con la profundidad. Hay una mayor preferencia de la mojabilidad por agua cerca de la base de la zona de transición, y una mayor preferencia de la mojabilidad por petróleo cerca del tope. En las zonas más altas se puede desestabilizar la película de agua, permitiendo que el petróleo contacte el sólido. En una posición más baja de la estructura, las superficies sólidas retienen en su mayoría la película de agua.

- Efecto de fluidos de perforación y completamiento en la mojabilidad. Los fluidos de perforación y workover, contienen surfactantes que pueden invadir los espacios porosos y alterar la mojabilidad en la región vecina al pozo. Una salmuera inyectada, cuyo contenido de sólidos disueltos o su $\mathrm{pH}$ difiere de los de la salmuera de formación, puede inducir cambios en la mojabilidad. Los surfactantes, incluyendo los generados por la acción microbiana, pueden reducir la tensión interfacial entre los fluidos y modificar el ángulo de contacto. El cuarzo tiende a volverse más mojable por petróleo a temperaturas más elevadas, pero la calcita tiende a volverse más mojable por agua. Por lo tanto, los métodos de recuperación termal pueden modificar la mojabilidad.

El pH y las condiciones de concentración para que exista una película de agua estable son diferentes para cada tipo de mineral dentro del espacio poroso (Abdallah, et al., 2011).

\section{Protocolo experimental}

El protocolo experimental se enfoca en tres grandes etapas (ver Figura 6):

- Etapa 1: Caracterización básica de fluidos de formación

- Etapa 2: Evaluación fluido-fluido y comportamiento reológico de fluidos de formación Vs Tratamientos químicos.

- Etapa 3: Evaluación del efecto sobre la humectabilidad: cualitativa en tapas de plug (ángulos de contacto) y desplazamientos en núcleos (evaluación permeabilidad relativa).

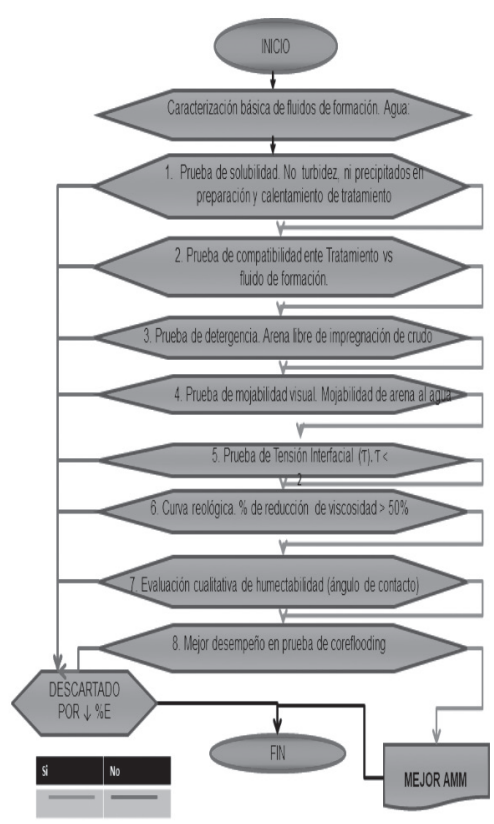

Figura 6. Protocolo experimental para evaluación de alteradores de humectabilidad. Fuente: Los Autores.

Cada etapa se describe de una manera detallada a continuación:

2.1. Etapa 1: caracterización básica de fluidos de formación.

Comprende el análisis fisicoquímico del agua de formación, la caracterización básica del crudo y la caracterización de las emulsiones.

- Caracterización físico química del agua de formación. El análisis fisicoquímico básico contempla: $\mathrm{pH}$, conductividad, alcalinidad, hierro disuelto y $\mathrm{CO} 2$. El $\mathrm{pH}$ y la conductividad se determinan con un pH-metro y conductímetro Horiba. La alcalinidad, HCO3- y CO3 se determinan por titulación potenciométrica (ASTM, 2001).

- Deshidratación del crudo muerto. Tanto la separación del agua emulsionada (Rondón, Bouriat, Lachaise \& Salager, 2006) como la deshidratación del crudo se realizara usando solo métodos físicos. Estos métodos corresponden a ultrasonido con calentamiento y centrifugación con calentamiento. La verificación del contenido de agua se realizara por el método Karl Fisher.

- Caracterización básica del crudo muerto: SARA, CSI, ${ }^{\circ}$ API, Viscosidad, Test de Oliensis. 
- Análisis SARA. La determinación de las fracciones del SARA de los crudos se realizara basado en cromatografía líquida de columna abierta, que permite cuantificar Saturados, Aromáticos, Resinas y Asfaltenos (Speight, 1984). Con los datos del análisis SARA se calculara el índice de estabilidad coloidal (CSI, Colloidal Stability Index) empleando la siguiente expresión:

$$
C S I=\frac{(\text { Saturadas }+ \text { Asfaltenos })}{(\text { Resinas }+ \text { Aromáticos })}
$$

Si CSI < 0.7: asfaltenos son estables.

Sí CSI > 0.9: asfaltenos son inestables.

Si CSI está entre 0.7 y 0.9 : el comportamiento es incierto (Mousavi, Riazi, Vafaie \& Mansoori, 2004).

- Porcentaje de contenido de agua. Para la determinación del porcentaje de contenido de agua, se seguirá el protocolo propuesto en la norma técnica ASTM D400708 para determinación de BSW (ASTM, 2008). Los valores se verificarán por el método de KarlFisher.

- Gravedad API ( ${ }^{\circ}$ API). La determinación de ${ }^{\circ}$ API en crudos pesados se realiza mediante el método del picnómetro según la norma ASTM D70, "Standard Test Method for Density of SemiSolid Bituminous Materials (ASTM, 2009).

- Test de oliensis. El procedimiento consiste en la adición al crudo de un alcano (n-heptano), con incrementos de un mililitro a una mezcla en agitación continua de 6 gramos de crudotolueno en relación 70-30. Después de cada adición de $\mathrm{n}$ - heptano, (agregados a intervalos de 1 minuto), se toma una gota de la mezcla y se deja caer sobre un papel filtro Whatman 1. Si los asfaltenos han floculado o aglomerado para formar agregados de mayor tamaño, se difunden más lentamente en el papel y la mancha exhibe una aureola central más o menos nítida según los casos. Si se continúa diluyendo n-heptano se puede observar que la aureola presenta una evolución desde una huella apenas discernible hasta un círculo negro de pequeño diámetro. El error de este método es menor del 1\% (Branco, Mansoori, De Almeida, 2001).

- Viscosidad de emulsiones. Se realiza una caracterización de las emulsiones presentes en los crudos para determinar los valores de viscosidad en función del contenido de agua, tasas de corte y temperatura.

2.2. Etapa 2: evaluación fluido- fluido y comportamiento reológico de fluidos de formación vs. Tratamientos químicos.

- Caracterización convencional de aditivos. La caracterización incluye los siguientes parámetros fisicoquímicos: $\mathrm{pH}$, densidad e índice de refracción. Adicionalmente se deben tomar espectros FTIR, los cuales se asimilan a una huella digital única para cada compuesto.

- Comportamiento reológico. Evaluación de los tratamientos químicos en un rango amplio de tasa de corte. La dosificación de los productos se fija en 1000 ppm con relación al volumen total. El efecto se evalúa sobre los crudos de los campos en estudio. Las mediciones se realizan a las temperaturas de 30 , $80{ }^{\circ} \mathrm{C}$ y Temperatura de yacimiento. Las tasas de corte están en el rango de 1 a 100 s-1.

- Evaluación de interacciones fluido-fluido y fluido-roca. Para cada producto se prepara una solución en salmuera sintética de los campos en estudio, con 2000 ppm como producto en la fase acuosa adicionada,

- Compatibilidad o interacción fluido-fluido. Se realiza a temperatura de yacimiento entre los fluidos de formación y los tratamientos químicos a evaluar (Molano, Navarro \& Díaz, 2014). Se realizan mezclas en proporciones $50-50$ y $80-$ 20 (crudo - tratamiento). Manualmente se agita por un minuto y se someten a calentamiento por dos (2) horas a la temperatura de yacimiento, se observa si hay separación de fases, formación de precipitados, rompimiento de emulsiones, calidad del agua separada y cambios aparentes de viscosidad (Bourrel, Salager, Schechter \& Wade, 1980).

- Mojabilidad visual. Se realiza con arena de formación o en su defecto utilizando arenas Ottawa 20/40 y en la misma se determina visualmente la tendencia que tiene un tratamiento a mojar una arena por agua o por crudo. Este análisis sigue la Norma API RP 42

- Tensión interfacial: La tensión interfacial es el resultado de efectos moleculares por los cuales se forma una interface o superficie que separa 
dos fluidos; si la tensión es nula, entonces se dice que los fluidos son miscibles entre sí. Para efectos de este estudio, se midieron las tensiones interfaciales a través de un tensiómetro que utiliza el método de la gota giratoria (API, 1977).

- Detergencia. En esta prueba se determina la capacidad de limpieza del surfactante disuelto en el tratamiento y la mojabilidad que induce el mismo (medida indirecta). En la Figura 7, se observan criterios de calificación.
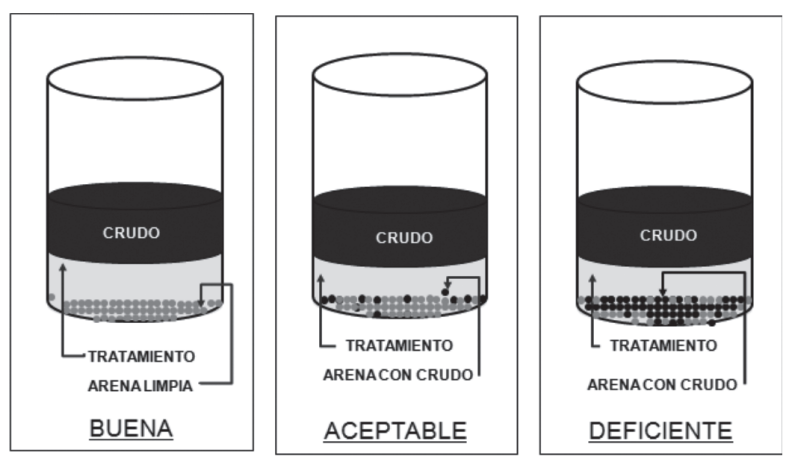

Figura 7. Apariencia y calificación de la prueba de detergencia. Fuente: Ecopetrol.

\subsection{Etapa 3: evaluación del efecto sobre la humectabilidad}

La dosificación de los productos se fija en $1.000 \mathrm{ppm}$ con relación al volumen total y los tratamientos son preparados con agua sintética equivalente al agua de formación de los campos en estudio. El efecto de la humectabilidad se evalúa en tapas de Plug y en núcleos.

- Evaluación cualitativa de humectabilidad en tapas de plugs. La medición de ángulos de contacto en tapas de plugs, se hace con una cámara digital de alta resolución y permite evaluar cambios de mojabilidad de la roca. Se empleará una pipeta para colocar una gota de líquido (hidrocarburo o aguasalmuera) en la superficie de la tapa del plug. La configuración de la gota sobre la tapa del plug se magnifica y se captura con una cámara digital y esta imagen es utilizada para estimar el ángulo de contacto generado entre la gota de fluido y la roca (Kwok, Neumann, 1999).

Las tapas de plug al ser tratadas con el químico, se someterán a un proceso de añejamiento a temperatura de yacimiento por un período entre 12 y 18 de horas, tiempo al final del cual se secarán por un periodo mínimo de 2 horas.
- Evaluación de permeabilidad relativa en pruebas de coreflooding. Se evalúa en plugs del campo en estudio la permeabilidad absoluta (Kabs) a la salmuera de formación, luego se desplaza crudo mineral hasta saturación irreducible de agua (Swirr) y evalúa la permeabilidad efectiva al crudo inicial (Keo) (API, 1998).

La muestra es luego sometida a restauración de Mojabilidad, con crudo deshidratado de los campos en estudio, durante quince días.

Después del proceso de restauración se desplaza nuevamente crudo muerto deshidratado para medición de la permeabilidad efectiva al crudo post-restauración de mojabilidad preferencial, la cual es la permeabilidad base para la prueba de permeabilidad relativa crudo-agua.

El desplazamiento de crudo se realiza hasta obtener condición de saturación irreducible de agua (Swirr), garantizando condiciones estables de flujo, presión, temperatura y tasa de inyección. Luego se hace el waterflooding a condiciones no estables, hasta obtener condición de saturación residual de crudo (sor) en la muestra. Se hace el monitoreo de efluentes vs. tiempo y presión vs. tiempo.

Al final de waterflooding se continúa desplazando salmuera hasta evaluar la permeabilidad efectiva al agua a condición de Sor. De esta manera se obtiene la línea base de permeabilidad relativa del plug del campo en estudio.

Posteriormente se inyecta el tratamiento y se deja en remojo por 24 horas, luego se desplaza crudo muerto deshidratado para llevar a saturación irreducible de agua y medir la permeabilidad efectiva al crudo post-tratamiento. Luego se realiza el segundo waterflooding para evaluar el efecto del tratamiento en la permeabilidad relativa al crudo y al agua.

El equipo de desplazamiento es un "loop" cerrado para recirculación de ambas fases a las condiciones de yacimiento, en estado no estable, una fase puede ser inyectada en el núcleo. Las condiciones límites de operación son: Temperatura $=270^{\circ} \mathrm{F}$, Presión de confinamiento $=6000$ psi y presión de poro $=5000$ psi.

En la Figura 8, se muestra un esquema del equipo experimental. El equipo consiste en: sistema 
temperatura, bombeo, porta núcleos, transmisores de presión diferencial y un separador acústico para medición de efluentes.

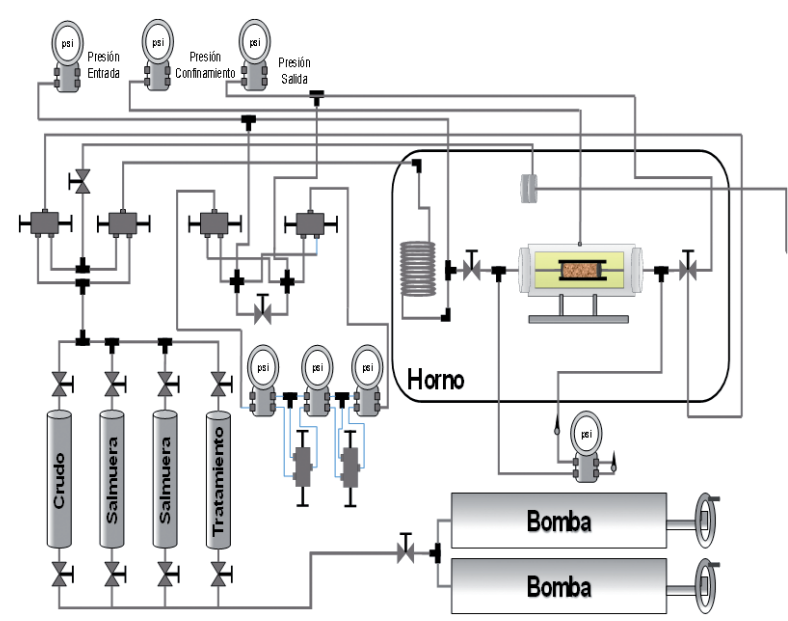

Figura 8. Esquema equipo de permeabilidades relativas. Fuente: Autores.

\section{Resultados}

\subsection{Implementación del protocolo o caso de estudio}

Para la implementación del protocolo experimental, se realizó un caso de estudio para evaluar tratamientos de ME y/o AMM base agua, con el objeto de identificar los mejores tratamientos que mejoren la humectabilidad de la roca hacia una mojabilidad al agua, que conduzca a un mejoramiento de la permeabilidad relativa al crudo (Kro) y a una disminución de la permeabilidad relativa al agua (Krw).
Se realizó un proceso de selección de sistemas químicos alteradores de humectabilidad de distintas compañías comerciales y empresas de servicios. Se suministró la información, los fluidos de formación y fuentes de agua de preparación de tratamientos del campo en estudio, para que cada empresa hiciera su respectiva evaluación experimental y optimizara el fluido que participaría en el proceso de evaluación de tratamientos de ME y/o AMM.

Para filtrar el proceso experimental a aquellas compañías que suministraran los mejores tratamientos, se realizó un pre-Screening básico de las compañías invitadas, ver tabla 1.

Tabla 1. Matriz evaluación pre-screening.

\begin{tabular}{|c|c|c|}
\hline \multicolumn{3}{|c|}{ Matriz evaluacion pre-screening } \\
\hline Evaluación & $\%$ peso & Observación \\
\hline $\begin{array}{l}\text { Tension interfacial - Método } \\
\text { Spinning Drop }\end{array}$ & 20 & Evaluación en el ICP \\
\hline Compatibilidades Vs Tiempo & 10 & Evaluación en el ICP \\
\hline Mojabilidades & 10 & Evaluación en el ICP \\
\hline $\begin{array}{l}\text { Curva Reologica (linea base y } \\
\text { Mezcla crudo + Tratamiento)** }\end{array}$ & 30 & Evaluación en el ICP \\
\hline $\begin{array}{l}\text { Evaluación cualitativa } \\
\text { humectabilidad con tapas de } \\
\text { plugs }\end{array}$ & 30 & Evaluación en el ICP \\
\hline TOTAL & 100 & \\
\hline
\end{tabular}

\subsection{Resultados de implementación del protocolo primera fase o pre-screening}

De acuerdo con el protocolo experimental y con la matriz de la tabla 1 , se realizó la evaluación del prescreening, el cual se presenta en la tabla 2.

Tabla 2. Resultados de implementación del protocolo para la fase pre-screening.

\begin{tabular}{|c|c|c|c|c|c|c|}
\hline \multicolumn{7}{|c|}{ Matriz evaluacion pre-screening } \\
\hline \multirow[b]{2}{*}{ Evaluación } & \multicolumn{6}{|c|}{ Compañía de Servicio Técnico (CST) } \\
\hline & $\mathbf{A}$ & $\mathbf{B}$ & C & $\mathbf{D}$ & $\mathbf{E}$ & $\mathbf{F}$ \\
\hline Solubilidad & OK & OK & OK & OK & OK & OK \\
\hline Compatibilidades Vs Tiempo & OK & OK & OK & NO & OK & OK \\
\hline Detergencia & OK & OK & OK & MALA & OK & OK \\
\hline Mojabilidades & CRUDO & AGUA & MIXTA & CRUDO & AGUA & AGUA \\
\hline Tension interfacial - Metodo Spinning Drop & 3,15 & 1,491 & 1,412 & N.R. & 1,315 & 1,499 \\
\hline $\begin{array}{l}\text { Curva Reologica (línea base y Mezcla crudo + Tratamiento, } \\
\% \text { Reducción de viscosidad) }\end{array}$ & $70 \%$ & $82 \%$ & $19 \%$ & $43 \%$ & $57 \%$ & $90 \%$ \\
\hline $\begin{array}{l}\text { Evaluación cualitativa humectabilidad con tapas de plug con } \\
\text { gota agua, variación ángulo de contacto }\end{array}$ & $81,4^{\circ} \rightarrow 0^{\circ}$ & $89,8^{\circ} \rightarrow 0^{\circ}$ & $83,3^{\circ} \rightarrow 0^{\circ}$ & $87,41 \rightarrow 0^{\circ}$ & $87,0^{\circ} \rightarrow 0^{\circ}$ & $83,2^{\circ} \rightarrow 0^{\circ}$ \\
\hline $\begin{array}{l}\text { Evaluación cualitativa humectabilidad con tapas de plug con } \\
\text { gota crudo, variación ángulo de contacto }\end{array}$ & $0^{\circ} \rightarrow 6,5^{\circ}$ & $0^{\circ} \rightarrow 10,9^{\circ}$ & $0^{\circ} \rightarrow 11,2^{\circ}$ & $0^{\circ} \rightarrow 0^{\circ}$ & $0^{\circ} \rightarrow 12,4^{\circ}$ & $0^{\circ} \rightarrow 6,7^{\circ}$ \\
\hline
\end{tabular}


De acuerdo con los pesos asignados para cada prueba y los resultados de la evaluación presentada en la tabla 2, se asignaron los puntajes respectivos para cada compañía, los cuales se muestran en la tabla 3.

En algunos casos se asignó el mayor puntaje al mayor valor de la prueba y los demás puntajes se aplicaron proporcionalmente, por ejemplo para el caso de reducción de la viscosidad, a mayor reducción de viscosidad del tratamiento, mayor puntaje. En otros casos el mayor puntaje se aplicó al menor valor de la prueba y los demás puntajes se aplicaron inversamente proporcional, por ejemplo para el caso de las tensiones interfaciales, a menor tensión interfacial entre el tratamiento y el crudo, mayor puntaje. En la prueba de compatibilidad se estableció dar el puntaje máximo de 10 puntos a la compañías que presentaron tratamientos con excelente separación, interface definida y sin manifestaciones de emulsiones e incompatibilidades como precipitados. Si existía una interface irregular o presencia de menisco, turbidez en fase acuosa, gotas de crudo en fase agua; se asignaba un puntaje de 8 . Para los tratamientos que presentaron una emulsión fuerte se calificó con 0 .

En el caso de la prueba de mojabilidad se asignó un puntaje de 10, 5 y 0 para tratamientos con mojabilidad al agua, mixta y al crudo respectivamente. En el caso de la evaluación cualitativa de la humectabilidad en tapas de plug, el puntaje se dividió en dos, 15 puntos para el efecto sobre el ángulo de contacto al agua y los otros 15 puntos para el efecto sobre el ángulo de contacto al crudo.

Tabla 3. Puntaje de las compañías en la evaluación de pre-Screening

\begin{tabular}{|l|c|c|c|c|c|c|c|}
\hline \multicolumn{1}{|c|}{ Matriz evaluacion pre-screening } \\
\hline \multicolumn{1}{|c|}{ Evaluación } & \% peso & \multicolumn{5}{c|}{ CST } \\
\cline { 5 - 9 } & & A & B & C & D & E & F \\
\hline Tension interfacial - Metodo Spinning Drop & 20 & 8,33 & 17,64 & 18,63 & 0 & 20,00 & 17,55 \\
\hline Compatibilidades Vs Tiempo & 10 & 8 & 10 & 10 & 0 & 8 & 8 \\
\hline Mojabilidades & 10 & 0 & 10 & 5 & 0 & 10 & 10 \\
\hline Curva Reologica (línea base y Mezcla crudo + Tratamiento)** & 30 & 23,33 & 27,41 & 6,42 & 14,43 & 18,89 & 30,00 \\
\hline Evaluación cualitativa humectabilidad con tapas de plugs & 30 & 22,90 & 28,26 & 28,55 & 15,00 & 30,00 & 23,14 \\
\hline TOTAL & 100 & 62,57 & 93,30 & 68,59 & 29,43 & 86,89 & 88,69 \\
\hline
\end{tabular}

\subsection{Resultados de implementación del protocolo fase pre-screening}

En la prueba de tensión interfacial y humectabilidad, los mejores tratamientos fueron los de las compañías B, E y F. En la prueba de comportamiento reológico, los mejores tratamientos fueron los de las compañías A, B, F. El tratamiento de la compañía A tuvo reducciones importantes en viscosidad, sin embargo los resultados en tensión interfacial no fueron los mejores. Una baja tensión interfacial está estrictamente ligada con una humectabilidad de la roca al agua. Según el puntaje final de la tabla 3, de acuerdo con los pesos establecidos para cada prueba, las compañías B, E y F sacaron los mayores puntajes y fueron seleccionadas para pasar a la siguiente fase de evaluación en pruebas de desplazamiento en núcleos.

En la tabla 4 se muestran los resultados de variación (después del tratamiento -antes del tratamiento) de los siguientes parámetros: Krw (\%) (End Point), Keo (mD), Kew (mD), Sor (\%), Swir (\%) y Np (\%).
Tabla 4. Evaluación en pruebas de desplazamiento en núcleos.

\begin{tabular}{|l|c|c|c|}
\hline \multicolumn{1}{|c|}{ Campo } & \multicolumn{3}{c|}{ Castilla } \\
\hline \multicolumn{1}{|c|}{ Compañía } & B & F & E \\
\hline \multicolumn{2}{|c|}{ Parámetro } & \multicolumn{3}{c|}{ Variación } \\
\hline Krw (\%) & $167 \%$ & $23 \%$ & $15 \%$ \\
\hline Keo (mD) & $-46 \%$ & $-39 \%$ & $13 \%$ \\
\hline Kew (mD) & $37 \%$ & $-16 \%$ & $22 \%$ \\
\hline Sor (\%) & $8 \%$ & $31 \%$ & $29 \%$ \\
\hline Sirr (\%) & $-31 \%$ & $-17 \%$ & $15 \%$ \\
\hline Np (\%) & $11 \%$ & $-23 \%$ & $-27 \%$ \\
\hline
\end{tabular}

De acuerdo con los resultados de la tabla 4, se asignaron los puntajes para la evaluación roca-fluido, los cuales se muestran en la tabla 5. 
Tabla 5. Puntaje de las compañías B, F y E en la evaluación en núcleos.

\begin{tabular}{|c|c|c|c|c|}
\hline \multicolumn{5}{|c|}{ Matriz de evaluación } \\
\hline \multirow{2}{*}{ Parámetro } & \multirow{2}{*}{$\%$ Peso } & \multicolumn{2}{l|}{ Compañía servicio técnico } \\
\cline { 3 - 5 } & & B & F & E \\
\hline Krw (\%) (End Point) & 10 & 0,91 & 6,47 & 10,00 \\
\hline Keo (mD) & 10 & 0,00 & 1,17 & 10,00 \\
\hline Kew (mD) & 10 & 2,81 & 10,00 & 3,53 \\
\hline Sor (\%) & 10 & 10,00 & 2,54 & 2,70 \\
\hline Swirr (\%) & 10 & 0,00 & 3,05 & 10,00 \\
\hline Np (\%) & 50 & 50,00 & 0,00 & 0,00 \\
\hline TOTAL & 100 & 63,7 & 23,2 & 36,2 \\
\hline
\end{tabular}

Con los puntajes obtenidos en las tablas $3(30 \%$ de peso) y tabla 5 ( $70 \%$ peso) se estableció un ranking final. En la tabla 6 se muestra el ranking final, siendo el tratamiento de mejor desempeño, el de la compañía B.

Tabla 6. Ranking final de evaluación de ME y/o AMM.

\begin{tabular}{|l|c|c|c|c|}
\hline \multirow{2}{*}{\multicolumn{1}{|c|}{ Evaluación }} & \multirow{2}{*}{ \% Peso } & \multicolumn{3}{|c|}{ Compañías servicio técnico } \\
\cline { 2 - 5 } & & B & F & E \\
\hline $\begin{array}{l}\text { Fluido-fluido, } \\
\text { comportamiento reológico }\end{array}$ & $30 \%$ & 93,3 & 88,69 & 86,89 \\
\hline Fluido-roca & $70 \%$ & 63,72 & 23,24 & 36,24 \\
\hline Puntaje total & $100 \%$ & 72,6 & 42,9 & 51,4 \\
\hline
\end{tabular}

\section{Discusión}

En las figuras 9 y 10 se presenta la comparación de las curvas $\mathrm{K}_{\text {rwo }}$ pre y post tratamiento para las compañías B y E, las cuales obtuvieron el mayor puntaje en el ranking final del BIC de microemulsiones. En ambas graficas se observa que hay desplazamiento de las curvas de permeabilidad relativa hacia la izquierda, lo cual indica una mayor tendencia de los tratamientos a mojar por crudo la roca, sin embargo, el tratamiento de la compañía B presentó un incremento en el $\mathrm{N}_{\mathrm{p}}$ del 11\%, lo cual se explica por la capacidad del tratamiento en reducir más la $\mathrm{S}_{\text {wir }}(31 \%)$ con respecto al incremento en el $\mathrm{S}_{\mathrm{or}}(8 \%)$, lo cual origina un incremento en el $\mathrm{N}_{\mathrm{p}}(\mathrm{Np}=$ $\left.1-\mathrm{S}_{\text {or }}-\mathrm{S}_{\text {wir }}\right)$. Lo anterior se evidenció en los volúmenes a la salida del separador, los cuales fueron cuantificados en dicha vasija.

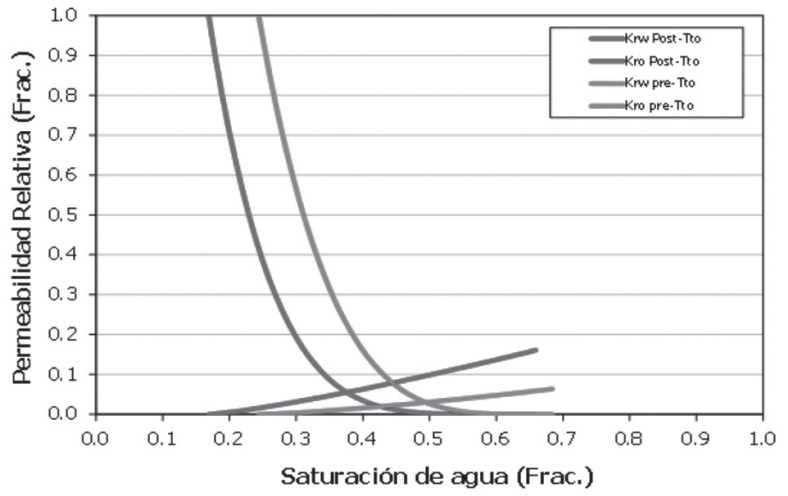

Figura 9. Comparación curvas Krwo, pre y post tratamiento $\mathrm{B}$.

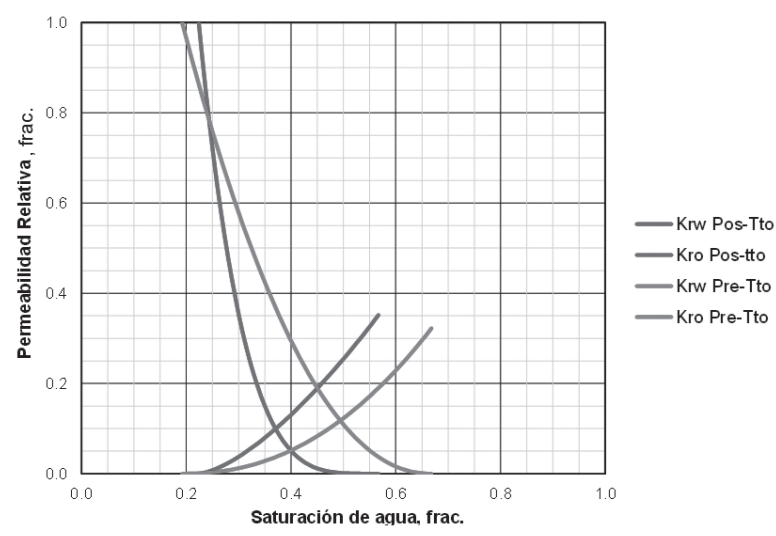

Figura 10. Comparación curvas Krwo, pre y post tratamiento $\mathrm{E}$.

\section{Conclusiones y recomendaciones}

1. El protocolo experimental desarrollado, es adecuado para la selección de un tratamiento óptimo para alterar la humectabilidad hacia una preferencia al agua.

2. El tratamiento de la compañía A tuvo reducciones importantes en viscosidad, sin embargo los resultados en tensión interfacial no fueron los mejores. Una baja tensión interfacial está estrictamente ligada con una humectabilidad de la roca al agua

3. De acuerdo con el ranking final de evaluación de microemulsiones y/o agentes mejoradores de movilidad, el tratamiento de la compañía B presentó el mejor desempeño.

4. A pesar de que el tratamiento de la compañía B presentó el mejor desempeño, se recomienda hacer una optimización del mismo para mejorar su 
eficiencia en la alteración de la humectabilidad del medio poroso.

5. El tratamiento de la compañía $B$ fue el único tratamiento que presentó un incremento en el $\mathrm{Np}$ (11\%), lo cual se explica por la capacidad del tratamiento en reducir más la Swir (31\%) con respecto al incremento en el Sor $(8 \%)$, lo cual origina un incremento en el $\mathrm{Np}(\mathrm{Np}=1$-Sor-Swir).

6. La naturaleza química de los fluidos de formación (petróleo, agua y gas), la tensión interfacial, la presión capilar, la superficie mineral, la temperatura, la presión de formación y la historia de saturación tienen incidencia sobre la mojabilidad del yacimiento.

\section{Agradecimientos}

Los autores agradecen al instituto colombiano del petróleo, ICP, por permitir la publicación de este artículo.

\section{Nomenclatura}

ACIPET: Asociación Colombiana de Ingenieros de Petróleos

ACP: Asociación Colombiana del Petróleo

AMM: Aditivos Mejoradores de Movilidad

ANH: Agencia Nacional de Hidrocarburos (Colombia)

CSI: Colloidal Stability Index

ICP: Instituto Colombiano del Petróleo

IEP: Informe de Estadísticas Petrolera (Colombia)

Krw: Permeabilidad relativa al agua

Kro: Permeabilidad relativa al crudo

Kew: Permeabilidad efectiva al agua

Keo: Permeabilidad efectiva al crudo

mD: Mili-Darcy

ME: Microemulsiones

SARA: Saturados, Arómaticos, Resinas y Asfaltenos

Sor: Saturación de crudo residual

Swir: Saturación de agua irreducible

Np: Factor de recobro

\section{Referencias bibliográficas}

1. Abdallah, W. et al. Fundamentals of Wettability, November 2011, Oilfield Review, volume 19, p. 51-56.

2. Asociación Colombiana del Petróleo. Informe Estadístico Petrolero 2015. Recuperado de https://acp.com.co/web2017/.../Informe\%20 Estadistico\%20Petrolero\%202014.pdf
3. ASTM D4007-08. "Standard Test Method for Water and Sediment in Crude Oil by the Centrifuge Method (Laboratory Procedure)". 2008.

4. ASTM D 4448. "Standard Guide for Sampling Ground-Water Monitoring Wells". 2001.

5. ASTM D70, "Standard Test Method for Density of Semi-Solid Bituminous Materials (Pycnometer Method)". 2009.

6. Bourrel M., Salager J., Schechter R. S., Wade W. H.A correlation for phase behavior of nonionic surfactants, J. Colloid Interf. Sci., 1980, 75, p. 451-461.

7. Branco V., Mansoori G., de Almeida L. Asphaltene flocculation and collapse from petroleum fluids. Journal Petroleum Science and Engineering, 2001, p. 217.

8.Camacho, R. P., \& Grosso, J. L. (2003). Criterios para la selección del sistema de levantamiento artificial para crudos pesados y extrapesados. Fuentes: El reventón energético, 3(1), 2.

9.Díaz, R. J., Navarro, S. F. M., \& Tavera, C. P. S. (2007). Modelo estadístico para la realización de analogías orientadas a procesos de recobro mejorado. Revista Fuentes, 5(1).

10. Figueira, J. N., Simão, R. A., Soares, B. G., \& Lucas, E. F. (2017). The influence of chemicals on asphaltenes precipitation: a comparison between atomic force microscopy and near infrared techniques. Revista Fuentes, 15(1), 7-17.

11. García, C. A., Rodríguez, O. C., Casallas, P. A., Cruz, G., Hernández, F. E., Afanador, L. E., \& Rodríguez, L. (2010). Optimización del transporte por oleoducto de crudo pesado Castilla. Fuentes: El reventón energético, $8(1), 2$.

12. Guerrero-Martin, C. A., Montes-Páez, E., de Oliveira, K., Cristina, M., Campos, J., \& Lucas, E. F. (2018, June). Calculating Asphaltenes Precipitation Onset Pressure by Using Cardanol as Precipitation Inhibitor: A Strategy to Increment the Oil Well Production. In SPE Trinidad and Tobago Section Energy Resources Conference. Society of Petroleum Engineers.

13. Jaimes M., Pachón Z., Villar A., Dorado, R. Technical/ Economic Selection and Evaluation of Chemical Treatments to Enhance the Efficiency of Artificial Lifting Systems in the Production of Heavy Crude: A Colombian Field Application. Heavy Oil Latin America Congress, 2011, p. 1-3. 
14. Kwok D. Y., Neumann, A. W. Contact angle measurement and contact angle interpretation. Adv. Colloid Interface Science, volume 81, 1999, p. 167-249.

15. Martin, C. A. G., \& Páez, E. M. (2017). Efeito da salinidade na tensão interfacial do sistema óleo/ agua em condições isobáricas e incremento gradual da temperatura. Revista Fuentes, 15(2), 117-124.

16. Martínez-Palou, R., de Lourdes Mosqueira, M., Zapata-Rendón, B., Mar-Juárez, E., BernalHuicochea, C., de la Cruz Clavel-López, J., \& Aburto, J. (2011). Transportation of heavy and extra-heavy crude oil by pipeline: A review. Journal of petroleum science and engineering, 75(3-4), 274-282.

17. Meneses, A. F. O., Moreno, L. F. C., \& Plata, J. A. R. (2017). Metodología experimental para la estimación de permeabilidades relativas en dos y tres fases por medio de ajuste histórico. Revista Fuentes, 15(1), 75-85.

18. Molano, A. M. J., Navarro, S. F. M., \& Díaz, R. J. (2014). Metodología para el diseño de baches en un proceso de inyección de polímeros para recobro mejorado, considerando fenómenos de interacción roca/fluidos. Fuentes: El reventón energético, 12(2), 6.

19. Mousavi-Dehghani, S. A., Riazi, M. R., VafaieSefti, M., \& Mansoori, G. A. (2004). An analysis of methods for determination of onsets of asphaltene phase separations. Journal of Petroleum Science and Engineering, 42(2-4), 145-156.

20. Norma API RP 40 "Recommended practices for core analysis”. American Petroleum Institute,
Washington D.C. Second Edition, 1998.

21. Norma API RP 42: "Laboratory Testing of Surface Active Agents for Well Stimulation, Section 3: Interfacial Tension and Wettability", American Petroleum Institute, Washington D.C. Second Edition, 1977.

22. Rondón, Miguel, et al. Breaking of water-incrude oil emulsions. 1. Physicochemical phenomenology of demulsifier action. Energy and Fuels. 20, p. 1600-1604, 2006.

23. Salager, J.L., Antón R., Aubry, J.M. Formulación de Micoremulsiones por el Método del HLD. Techniques de I'Inegénieur, Vol. Génie des Procédés, 2001, artículo J2 157, 1-20.

24. Sanchez, A. F. P., Sanchez, J. D. B., Gonzalez, J. F. R., \& Ramirez, L. E. M. (2017). Transporte de crudo pesado por oleoducto usando el método de dilución: Un enfoque práctico para modelar la caída de presión y la precipitación de asfaltenos. Revista Fuentes, 15(2), 7-17.

25. Sierra, D. M., Navarro, S. F. M., \& Tavera, C. P. S. (2006). Simulación de un piloto de inyección continua de vapor usando pozos horizontales. Fuentes: El reventón energético, 4(2), 2.

26. Speight, J., "Characterization of Heavy Crude Oils and Petroleum Residues," Symposium International, Editions Technip, Paris, 1984, pp. $32-41$.

27. Trujillo, M., Delgadillo, C., Claro, Y., Rojas, R., \& Sandoval, J. (2018). Reduciendo la incertidumbre en la ejecución de un piloto de combustión in situ en un campo de crudo extra pesado colombiano mediante la realización de una prueba de conectividad con nitrógeno. Revista Fuentes, 16(2).

Recepción: 20 de noviembre de 2018

Aceptación: 12 de diciembre de 2019 